\title{
NEARLY HYPERHARMONIC FUNCTIONS AND JENSEN MEASURES
}

\author{
Wolfhard Hansen and Ivan Netuka \\ Universität Bielefeld, Fakultät für Mathematik \\ 33501 Bielefeld, Germany; hansen@math.uni-bielefeld.de \\ Charles University, Faculty of Mathematics and Physics, Mathematical Institute \\ Sokolovská 83, 18675 Praha 8, Czech Republic; netuka@karlin.mff.cuni.cz
}

\begin{abstract}
Let $(X, \mathcal{H})$ be a $\mathcal{P}$-harmonic space and assume for simplicity that constants are harmonic. Given a numerical function $\varphi$ on $X$ which is locally lower bounded, let

$$
J_{\varphi}(x):=\sup \left\{\int^{*} \varphi d \mu: \mu \in \mathcal{J}_{x}(X)\right\}, \quad x \in X,
$$

where $\mathcal{J}_{x}(X)$ denotes the set of all Jensen measures $\mu$ for $x$, that is, $\mu$ is a compactly supported measure on $X$ satisfying $\int u d \mu \leq u(x)$ for every hyperharmonic function $u$ on $X$. The main purpose of the paper is to show that, assuming quasi-universal measurability of $\varphi$, the function $J_{\varphi}$ is the smallest nearly hyperharmonic function majorizing $\varphi$ and that $J_{\varphi}=\varphi \vee \hat{J}_{\varphi}$, where $\hat{J}_{\varphi}$ is the lower semicontinuous regularization of $J_{\varphi}$. So, in particular, $J_{\varphi}$ turns out to be at least "as measurable as" $\varphi$. This improves recent results, where the axiom of polarity was assumed. The preliminaries on nearly hyperharmonic functions in the framework of balayage spaces are closely related to the study of strongly supermedian functions triggered by Mertens more than forty years ago.
\end{abstract}

\section{Representing measures for positive hyperharmonic functions}

Let $X$ be a locally compact space with countable base, let $\mathcal{B}$ denote the $\sigma$-algebra of all Borel sets in $X$, and let $\mathcal{B}(X), \mathcal{C}(X)$ respectively be the set of all numerical functions on $X$ which are Borel measurable, continuous and real respectively. Further, let $\mathcal{W}$ be a convex cone of positive lower semicontinuous numerical functions on $X$ having the following properties.

(C) Continuity: Every $w \in \mathcal{W}$ is the supremum of its minorants in $\mathcal{W} \cap \mathcal{C}(X)$.

$(\mathrm{S})$ Separation: $\mathcal{W}$ is linearly separating, that is, for all $x \neq y$ and $\gamma>0$, there exists a function $v \in \mathcal{W}$ such that $v(x) \neq \gamma v(y)$.

(T) Transience: There are strictly positive functions $u, v \in \mathcal{W} \cap \mathcal{C}(X)$ such that $u / v$ tends to 0 at infinity.

The fine topology on $X$ is the coarsest topology on $X$ which is at least as fine as the initial topology and such that every function in $\mathcal{W}$ is continuous. Given a numerical function $g$ on $X$, let $\hat{g}, \hat{g}^{f}$ respectively denote the largest lower semicontinuous, finely lower semicontinuous respectively minorant of $g$.

We recall that $(X, \mathcal{W})$ is a balayage space if the following hold:

- $\mathcal{W}$ has the properties $(\mathrm{C}),(\mathrm{S})$ and $(\mathrm{T})$.

- If $v_{n} \in \mathcal{W}, v_{n} \uparrow v$, then $v \in \mathcal{W}$.

- If $\mathcal{V} \subset \mathcal{W}$, then $\widehat{\inf \mathcal{V}}^{f} \in \mathcal{W}$.

https://doi.org/10.5186/aasfm.2019.4401

2010 Mathematics Subject Classification: Primary 31B05, 31D05, 60J45, 60J75.

Key words: Jensen measure, nearly hyperharmonic function, strongly supermedian function. 
- If $u, v^{\prime}, v^{\prime \prime} \in \mathcal{W}, u \leq v^{\prime}+v^{\prime \prime}$, then there exist $u^{\prime}, u^{\prime \prime} \in \mathcal{W}$ such that $u=u^{\prime}+u^{\prime \prime}$ and $u^{\prime} \leq v^{\prime}, u^{\prime \prime} \leq v^{\prime \prime}$.

See $[4,11]$ for an exposition of the theory in all details; for a description, which is more expanded than the one given here, see [14, Appendix 8.1].

Examples 1.1. 1. If $(X, \mathcal{H})$ is a $\mathcal{P}$-harmonic space and ${ }^{*} \mathcal{H}^{+}(X)$ is the convex cone of positive hyperharmonic functions on $X$, then $\left(X,{ }^{*} \mathcal{H}^{+}(X)\right)$ is a balayage space. In particular, the theory of balayage spaces covers the potential theory for large classes of elliptic and parabolic differential equations of second order (for details the reader might consult [9, Section 7.1]). In addition, it covers, for example, the theory of Riesz potentials.

2. If $\mathfrak{X}$ is a Hunt process on $X$ with transition semigroup $\mathbf{P}=\left(P_{t}\right)_{t>0}$ such that its convex cone

$$
E_{\mathfrak{X}}:=\left\{u \in \mathcal{B}^{+}(X): \sup _{t>0} P_{t} u=u\right\}
$$

of excessive functions satisfies $(\mathrm{C}),(\mathrm{S})$ and $(\mathrm{T})$, then $\left(X, E_{\mathbf{P}}\right)$ is a balayage space. It is easily seen that $E_{\mathbf{P}}$ satisfies (C), if the resolvent kernels $V_{\lambda}:=\int e^{-\lambda t} P_{t} d t, \lambda>0$, are strong Feller, that is, $V_{\lambda} f \in \mathcal{C}(X)$ for every bounded $f \in \mathcal{B}(X)$.

We observe that, conversely, for every balayage space $(X, \mathcal{W})$ with $1 \in \mathcal{W}$, there exists a Hunt process $\mathfrak{X}$ on $X$ such that $E_{\mathfrak{X}}=\mathcal{W}$.

In the following let $(X, \mathcal{W})$ be a balayage space and let us fix a strictly positive function $u_{0} \in \mathcal{W} \cap \mathcal{C}(X)$. Further, let $\mathcal{P}(X)$ be the set of all continuous real potentials, that is, functions $p \in \mathcal{W} \cap \mathcal{C}(X)$ such that $p / v$ vanishes at infinity for some strictly positive $v \in \mathcal{W} \cap \mathcal{C}(X)$. We shall say that a numerical function $\varphi$ on $X$ is $\mathcal{P}$-bounded, if $|\varphi| \leq p$ for some $p \in \mathcal{P}(X)$; the set of all $\mathcal{P}$-bounded functions in $\mathcal{C}(X)$ will be denoted by $\mathcal{C}_{\mathcal{P}}(X)$. For every numerical function $\varphi$ on $X$, let $\hat{\varphi}$ denote its lower semicontinuous regularization, that is, $\hat{\varphi}(x):=\liminf _{y \rightarrow x} \varphi(y)$ for every $x \in X$. If $\mathcal{V}$ is a subset of $\mathcal{W}$ and $v:=\inf \mathcal{V}$, then $\hat{v} \in \mathcal{W}$ and $\hat{v}(x)=\hat{v}^{f}(x):=\mathrm{f}-\lim _{\inf }{ }_{y \rightarrow x} v(y)$, $x \in X$ (lower limit with respect to the fine topology).

We recall that, for every numerical function $\varphi \geq 0$, a reduced function $R_{\varphi}$ and a swept function $\hat{R}_{\varphi}$ are defined by

$$
R_{\varphi}:=\inf \{u \in \mathcal{W}: u \geq \varphi\} \quad \text { and } \quad \hat{R}_{\varphi}:=\widehat{R_{\varphi}} .
$$

In particular, we have $R_{v}^{A}:=R_{v 1_{A}} \leq v$ and $\hat{R}_{v}^{A}:=\hat{R}_{v 1_{A}} \leq R_{v}^{A}$ for $A \subset X$ and $v \in \mathcal{W}$, which leads to reduced measures $\varepsilon_{x}^{A}$ and swept measures $\hat{\varepsilon}_{x}^{A}, x \in X$, characterized by $\int v d \varepsilon_{x}^{A}=R_{v}^{A}(x)$ and $\int v d \hat{\varepsilon}_{x}^{A}=\hat{R}_{v}^{A}(x), v \in \mathcal{W}$. Let us observe that $\varepsilon_{x}^{A}=\hat{\varepsilon}_{x}^{A}$ for every $x \in A^{c}$, since $\hat{R}_{v}^{A}=R_{v}^{A}$ on $A^{c}$ (see [4, VI.2.4]). If $x \in A$, then $\varepsilon_{x}^{A}=\varepsilon_{x}$ and, by [4, VI.9.2], $\hat{\varepsilon}_{x}^{A}=\hat{\varepsilon}_{x}^{A}(\{x\}) \varepsilon_{x}+\left(1-\hat{\varepsilon}_{x}^{A}(\{x\}) \varepsilon_{x}^{A \backslash\{x\}}\right.$.

For every $x \in X$, let $\mathcal{M}_{x}(\mathcal{W})$ denote the convex set of all representing measures for $x$ with respect to $\mathcal{W}$, that is, (positive Radon) measures $\mu$ on $X$ such that, for every $w \in \mathcal{W}$,

$$
\int w d \mu \leq w(x)
$$

Since every function in $\mathcal{W}$ is an increasing limit of a sequence in $\mathcal{P}(X),(1.2)$ holds for functions in $\mathcal{W}$, if it holds for functions in $\mathcal{P}(X)$. Let $\mathcal{B}^{*}$ denote the $\sigma$-algebra of all $(\mathcal{B}$-)universally measurable sets in $X$. By [4, VI.12.5, 2.2, 4.3, 4.4],

$$
E:=\left\{\varepsilon_{x}^{A}: A \subset X\right\}=\left\{\varepsilon_{x}\right\} \cup\left\{\varepsilon_{x}^{A}: A \in \mathcal{B}, A \text { finely closed, } x \notin A\right\}
$$


is the set of extreme points of $\mathcal{M}_{x}(\mathcal{W})$. The set $\mathcal{M}_{x}(\mathcal{W})$ is weak ${ }^{*}$-compact, that is, for every sequence $\left(\mu_{m}\right)$ in $\mathcal{M}_{x}(\mathcal{W})$, there exists a subsequence $\left(\mu_{m_{k}}\right)$ and $\mu \in \mathcal{M}_{x}(\mathcal{W})$ such that $\lim _{k \rightarrow \infty} \int f d \mu_{m_{k}}=\int f d \mu$ for every $f \in \mathcal{C}_{\mathcal{P}}(X)$ (see [4, VI.10.1]). So we know by Choquet's theorem that, for every $\mu \in \mathcal{M}_{x}(\mathcal{W})$, there exists a probability measure $\rho$ on $E$ such that, for every $f \in \mathcal{C}_{\mathcal{P}}(X)$,

$$
\int f d \mu=\int\left(\int f d \nu\right) d \rho(\nu)
$$

and then (1.4) holds for every Borel measurable function $f \geq 0$ on $X$. (We might note that, for a given $\mu$, the measure $\rho$ does not have to be unique; see [12]).

By definition, a subset $P$ of $X$ is polar if $\hat{R}_{u_{0}}^{P}=0$. Every polar set $P$ is contained in a polar set in $\mathcal{B}$ (see $[4, \mathrm{VI} .2 .2])$. Let $\tilde{\mathcal{B}}, \widetilde{\mathcal{B}^{*}}$ respectively denote the $\sigma$-algebra of all sets $A$ in $X$ for which there exists a set $B$ in $\mathcal{B}, \mathcal{B}^{*}$ respectively such that the symmetric difference $A \triangle B$ is polar. We note that $\tilde{\mathcal{B}} \cap \mathcal{B}^{*}$ is the $\sigma$-algebra $\mathcal{B}^{n}$ of all nearly Borel sets in $X$ (see [3, page 57]).

If $\mu \in \mathcal{M}_{x}(\mathcal{W}), x \in X$, then $\mu$ does not charge polar sets $P$ in $X \backslash\{x\}$. Indeed, given $\varepsilon>0$, there exists a function $w \in \mathcal{W}$ such that $w=u_{0}$ on $P$ and $w(x)<\varepsilon$, and we have $\int_{P}^{*} u_{0} d \mu \leq \int w d \mu \leq w(x)<\varepsilon$ (cf. [5, Corollary 1.8]), whence $\mu^{*}(P)=0$. So we know that $\widetilde{\mathcal{B}^{*}}$ is contained in the completions of $\mathcal{B}$ with respect to the measures $\mu \in \mathcal{M}_{x}(\mathcal{W}), x \in X$.

\section{Positive nearly hyperharmonic functions}

Let $\mathcal{U}_{c}$ denote the family of relatively compact open sets in $X$ and $V^{c}:=X \backslash V$, $V \in \mathcal{U}_{c}$. We first recall that $\mathcal{W}$ is the set of positive hyperharmonic functions on $X$, that is, $\mathcal{W}$ consists of all lower semicontinuous numerical functions $u \geq 0$ on $X$ satisfying

$$
\int u d \varepsilon_{x}^{V^{c}} \leq u(x) \text { for all } x \in V \in \mathcal{U}_{c}
$$

(see [4, III.2.1]). Extending the definition given for harmonic spaces in [1, Section II.1] and [6, p. 119], let us say that an arbitrary function $u: X \rightarrow[0, \infty]$ is nearly hyperharmonic provided

$$
\int^{*} u d \varepsilon_{x}^{V^{c}} \leq u(x) \text { for all } V \in \mathcal{U}_{c} \text { and } x \in V
$$

where we may replace upper integrals by integrals, if $u$ is $\widetilde{\mathcal{B}^{*}}$-measurable.

Obviously, the following holds.

Proposition 2.1. The set $\mathcal{N}^{+}$of all positive nearly hyperharmonic functions on $X$ is a convex cone which contains $\mathcal{W}$. It is closed under increasing limits and, in contrast to $\mathcal{W}$, closed under arbitrary infima.

Let us note that, for all $V \in \mathcal{U}_{c}$ and $\varphi: X \rightarrow[0, \infty]$, the function

$$
H_{V}^{*} \varphi: x \mapsto \int^{*} \varphi d \varepsilon_{x}^{V^{c}}
$$

is lower semicontinuous on V; see [4, III.3.4] (it is even harmonic on $V$, if $\varphi \leq w$ for some $w \in \mathcal{W} \cap \mathcal{C}(X)$, a consequence of [4, VI.2.6]). This implies the following.

Proposition 2.2. Let $u \in \mathcal{N}^{+}$. Then $v \in \mathcal{N}^{+}$for every function $v$ satisfying $\hat{u} \leq v \leq u$. In particular, $\hat{u} \in \mathcal{W}$. 
Proof. Indeed, if $V \in \mathcal{U}_{c}$, then $H_{V}^{*} u \leq u$, whence $H_{V}^{*} v \leq H_{V}^{*} u \leq \hat{u} \leq v$ on $V$.

Moreover, by [4, III.6.14], for all $u \in \mathcal{N}^{+}$and $x \in X$,

$$
\hat{u}(x)=\hat{u}^{f}(x)=\sup \left\{\int^{*} u d \varepsilon_{x}^{V^{c}}: x \in V \in \mathcal{U}_{c}\right\}
$$

and, if $x \in X$ is not isolated,

$$
\hat{u}(x)=\lim _{V \downarrow\{x\}}\left\{\int^{*} u d \varepsilon_{x}^{V^{c}}: x \in V \in \mathcal{U}_{c}\right\} .
$$

Of course, $\hat{u}(x)=\hat{u}^{f}(x)=u(x)$ for isolated points $x$ in $X$. Hence we have the following (cf. [1, p. 48]).

Proposition 2.3. (i) For all $u, v \in \mathcal{N}^{+}, \widehat{u+v}=\hat{u}+\hat{v}$.

(ii) If $\left(u_{m}\right)$ is a sequence in $\mathcal{N}^{+}$and $u_{m} \uparrow u$, then $u \in \mathcal{N}^{+}$and $\hat{u}_{m} \uparrow \hat{u}$.

Given $\varphi: X \rightarrow[0, \infty]$, let

$$
N_{\varphi}:=\inf \left\{u \in \mathcal{N}^{+}: u \geq \varphi\right\}
$$

so that $N_{\varphi}$ is the smallest nearly hyperharmonic majorant of $\varphi$. Clearly,

$$
N_{\varphi} \leq R_{\varphi}
$$

since $\mathcal{W} \subset \mathcal{N}^{+}$. Further we easily obtain the following, where $f \vee g$ for functions $f, g$ on $\mathrm{X}$ denotes the pointwise maximum of $f$ and $g$.

Proposition 2.4. Let $\varphi: X \rightarrow[0, \infty]$ and $\hat{N}_{\varphi}:=\widehat{N}_{\varphi}$.

(i) Then $N_{\varphi}=\varphi \vee \hat{N}_{\varphi}$.

(ii) If $\varphi$ is finely lower semicontinuous, then $N_{\varphi}=\hat{N}_{\varphi}=R_{\varphi} \in \mathcal{W}$.

(iii) If $\varphi_{1}, \varphi_{2}, \ldots: X \rightarrow[0, \infty]$ and $\varphi_{m} \uparrow \varphi$, then $N_{\varphi_{m}} \uparrow N_{\varphi}$ and $\hat{N}_{\varphi_{m}} \uparrow \hat{N}_{\varphi}$.

Proof. (i) Clearly, $v:=\varphi \vee \hat{N}_{\varphi}$ satisfies $\hat{N}_{\varphi} \leq v \leq N_{\varphi}$. Therefore $v \in \mathcal{N}^{+}$, by Proposition 2.2, and $N_{\varphi} \leq v$ because of $\varphi \leq v$.

(ii) If $\varphi$ is finely lower semicontinuous, then $w:=\hat{N}_{\varphi} \in \mathcal{W}$ and $w \geq \varphi$, by (2.4). So $w \geq R_{\varphi} \geq N_{\varphi} \geq w$.

(iii) Consequence of Proposition 2.3,(ii) and (2.4).

For all functions $\varphi: X \rightarrow[0, \infty]$ and $x \in X$, let

$$
\begin{aligned}
& M_{\varphi}(x):=\sup \left\{\int^{*} \varphi d \mu: \mu \in \mathcal{M}_{x}(\mathcal{W})\right\}, \\
& M_{\varphi}^{\prime}(x):=\sup \left\{\int^{*} \varphi d \varepsilon_{x}^{V^{c}}: x \in V \in \mathcal{U}_{c}\right\}, \\
& M_{\varphi}^{\prime \prime}(x):=\sup \left\{\int^{*} \varphi d \varepsilon_{x}^{K}: K \text { compact in } X \backslash\{x\}\right\}, \\
& M_{\varphi}^{\prime \prime \prime}(x):=\sup \left\{\int^{*} \varphi d \varepsilon_{x}^{A}: A \in \mathcal{B}, A \text { finely closed, } x \notin A\right\},
\end{aligned}
$$

where we may replace the upper integrals by integrals, if $\varphi$ is $\widetilde{\mathcal{B}^{*}}$-measurable.

Proposition 2.5. Let $\varphi$ be a positive numerical function on $X$. Then

$$
M_{\varphi}^{\prime}=M_{\varphi}^{\prime \prime}=M_{\varphi}^{\prime \prime \prime} \leq M_{\varphi}
$$

If $\varphi$ is $\widetilde{\mathcal{B}^{*}}$-measurable, then $\varphi \vee M_{\varphi}^{\prime}=M_{\varphi}$. 
Proof. Of course, $M_{\varphi}^{\prime} \vee M_{\varphi}^{\prime \prime} \leq M_{\varphi}^{\prime \prime \prime} \leq M_{\varphi}$. Let us fix $x \in X$.

Let $A$ be a finely closed Borel set, $x \notin A$, and $b<\int^{*} \varphi d \varepsilon_{x}^{A}$. By [4, VI.4.6], $\varepsilon_{x}^{A}$ is supported by $A$. So there exists a compact $K$ in $A$ such that $\int^{*} 1_{K} \varphi d \varepsilon_{x}^{A}>b$. By [4, VI.9.4], $1_{K} \varepsilon_{x}^{A} \leq \varepsilon_{x}^{K}$, hence $b<\int^{*} 1_{K} \varphi d \varepsilon_{x}^{A} \leq \int^{*} \varphi d \varepsilon_{x}^{K} \leq M_{\varphi}^{\prime \prime}(x)$. Thus $M_{\varphi}^{\prime \prime \prime}(x) \leq M_{\varphi}^{\prime \prime}(x)$.

Next let $K$ be a compact set in $X \backslash\{x\}, b<c<\int^{*} \varphi d \varepsilon_{x}^{K}$ and $p \in \mathcal{P}(X)$, $p>0$. Then there exists $m \in \mathbf{N}$ such that $\varphi^{\prime}:=\varphi \wedge(m p)$ satisfies $\int^{*} \varphi^{\prime} d \varepsilon_{x}^{K}>c$. Since $q:=m p$ is a potential, there exists, by [4, II.5.2], a relatively compact open neighborhood $U$ of $\{x\} \cup K$ such that $\int q d \varepsilon_{x}^{U^{c}}<c-b$. Let us define $V:=U \backslash K$ and $\nu:=1_{U^{c}} \varepsilon_{x}^{V^{c}}$. By [4, VI.9.4],

$$
\nu \leq \varepsilon_{x}^{U^{c}} \quad \text { and } \quad \varepsilon_{x}^{K}=1_{K} \varepsilon_{x}^{V^{c}}+\nu^{K} .
$$

Therefore

$$
\int q d \nu^{K} \leq \int q d \nu \leq \int q d \varepsilon_{x}^{U^{c}}<c-b \text { and } c<\int^{*} \varphi^{\prime} d \varepsilon_{x}^{K} \leq \int^{*} \varphi d \varepsilon_{x}^{V^{c}}+\int q d \nu^{K} .
$$

So $b<\int^{*} \varphi d \varepsilon_{x}^{V^{c}}$, and we conclude that $M_{\varphi}^{\prime \prime}(x) \leq M_{\varphi}^{\prime}(x)$ completing the proof of the equalities in (2.8).

Finally, we suppose that $\varphi$ is $\widetilde{\mathcal{B}^{*}}$-measurable and fix $\mu \in \mathcal{M}_{x}(\mathcal{W})$. Let us assume for the moment that $\int \varphi d \mu<\infty$. There exist positive Borel measurable functions $f, g$ on $X$ such that $f \leq \varphi \leq g$ and

$$
\int f d \mu=\int \varphi d \mu=\int g d \mu .
$$

Using the integral representation (1.4), we see that $\int f d \nu=\int g d \nu$ for $\rho$-a.e. $\nu \in E$, and hence

$$
\int f d \nu=\int \varphi d \nu \leq \varphi(x) \vee M_{\varphi}^{\prime \prime \prime}(x) \text { for } \rho \text {-a.e. } \nu \in E \text {. }
$$

Thus $\int \varphi d \mu=\int f d \mu \leq \varphi(x) \vee M_{\varphi}^{\prime \prime \prime}(x)$, by (1.4) and (2.9). In the general case, we apply the previous considerations to the functions $\varphi \wedge\left(m u_{0}\right), m \in \mathbf{N}$, and let $m \rightarrow \infty$.

Corollary 2.6. Let $u$ be a positive numerical function on $X$ and $x \in X$. Then the following properties are equivalent:

(i) For every $V \in \mathcal{U}_{c}$ containing $x, \int^{*} u d \varepsilon_{x}^{V^{c}} \leq u(x)$.

(ii) For every subset $A$ of $X \backslash\{x\}, \int^{*} u d \varepsilon_{x}^{A} \leq u(x)$.

(iii) For every compact $K$ in $X \backslash\{x\}, \int^{*} u d \varepsilon_{x}^{K} \leq u(x)$.

If $u$ is $\widetilde{\mathcal{B}^{*}}$-measurable, then these properties hold if and only if $\int u d \mu \leq u(x)$ for every $\mu \in \mathcal{M}_{x}(\mathcal{W})$.

Moreover, we have the following.

Proposition 2.7. Let $u \in \mathcal{N}^{+}$be $\mathcal{B}^{*}$-measurable and let $\mu, \nu$ be measures on $X$ such that $\int w d \mu \leq \int w d \nu$ for every $w \in \mathcal{W}$, and $\int p d \nu<\infty$ for some strictly positive $p \in \mathcal{P}(X)$. Then $\int u d \mu \leq \int u d \nu$.

Proof. By [4, VI.12.6], there exists a kernel $V$ on $X$ such that $V(x, \cdot) \in \mathcal{M}_{x}(\mathcal{W})$ for every $x \in X$ and $\mu=\nu V$. By Corollary 2.6, $V u \leq u$. Let $f$ be a $\mathcal{B}$-measurable function on $X$ such that $0 \leq f \leq u$ and $f=u(\nu+\mu)$-a.e. Then $\int u d \mu=\int f d \mu=$ $\int V f d \nu$, where $V f \leq V u \leq u$. Thus $\int u d \mu \leq \int u d \nu$. 
So our (positive) nearly hyperharmonic functions are functions which in $[2,3,7$, $8,17,19]$ (mostly assuming additional measurability properties) are called strongly supermedian.

\section{Identity of $M_{\varphi}$ and $N_{\varphi}$}

In this section, we shall give a fairly straightforward proof for the following result.

Theorem 3.1. For every $\widetilde{\mathcal{B}^{*}}$-measurable numerical function $\varphi \geq 0$ on $X$,

$$
M_{\varphi}=N_{\varphi}=\varphi \vee \hat{N}_{\varphi} .
$$

In particular, $M_{\varphi}$ is the smallest nearly hyperharmonic majorant of $\varphi$, and $M_{\varphi}$ is (at least) "as measurable as $\varphi$ ", that is, if $\mathcal{A}$ is any $\sigma$-algebra on $X$ such that $\mathcal{B} \subset \mathcal{A} \subset \widetilde{\mathcal{B}^{*}}$ and $\varphi$ is $\mathcal{A}$-measurable, then $M_{\varphi}$ is $\mathcal{A}$-measurable.

Remark 3.2. In a more general setting, this has been shown by more involved methods for the smaller class of functions $\varphi \geq 0$ which are nearly Borel measurable or, slightly more general, nearly analytic (see [16, 8, 2, 3]).

To prove Theorem 3.1 we start considering the case, where $\varphi$ is upper semicontinuous and $\mathcal{P}$-bounded. We first recall the following ([11, Corollary 1.2.2]).

Proposition 3.3. For all upper semicontinuous $\mathcal{P}$-bounded positive functions $\psi, \psi_{1}, \psi_{2}, \ldots$ on $X$ the following holds:

- The function $R_{\psi}$ is upper semicontinuous. It is harmonic on $X \backslash \operatorname{supp}(\psi)$.

- If $\psi$ is continuous, then $R_{\psi}$ is continuous.

- If $\psi_{m} \downarrow \psi$, then $R_{\psi_{m}} \downarrow R_{\psi}$.

The following consequence of the theorem of Hahn-Banach is known in more general situations (see e.g. [18, p. 226]). For the convenience of the reader we include a complete proof.

Proposition 3.4. Let $\psi \geq 0$ be upper semicontinuous and $\mathcal{P}$-bounded. Then, for every $x \in X$, there exists $\mu \in \mathcal{M}_{x}(\mathcal{W})$ such that $\int \psi d \mu=R_{\psi}(x)$.

Proof. (a) Let $x \in X$ and $\varphi \in \mathcal{C}_{\mathcal{P}}(X), \varphi \geq 0$. Since the mapping $f \mapsto R_{f^{+}}(x)$ is sublinear on $\mathcal{C}_{\mathcal{P}}(X)$, there exists a linear form $\mu$ on $\mathcal{C}_{\mathcal{P}}(X)$ such that

$$
\mu(\varphi)=R_{\varphi}(x) \text { and } \mu(f) \leq R_{f^{+}}(x) \text { for every } f \in \mathcal{C}_{\mathcal{P}}(X) .
$$

If $f \in \mathcal{C}_{\mathcal{P}}(X)$ and $f \leq 0$, then $\mu(f) \leq R_{0}(x)=0$. Therefore $\mu$ is a measure on $X$. Of course, $\mu(p) \leq p(x)$ for every $p \in \mathcal{P}(X)$, and hence $\mu \in \mathcal{M}_{x}(\mathcal{W})$.

(b) There exist $\varphi_{m} \in \mathcal{C}_{\mathcal{P}}(X)$ such that $\varphi_{m} \downarrow \psi$. By (a), for every $m \in \mathbf{N}$, there exists a measure $\mu_{m} \in \mathcal{M}_{x}(\mathcal{W})$ such that $\mu_{m}\left(\varphi_{m}\right)=R_{\varphi_{m}}(x)$. We may (passing to a subsequence) assume without loss of generality that the sequence $\left(\mu_{m}\right)$ converges to a measure $\mu \in \mathcal{M}_{x}(\mathcal{W})$ (that is, $\lim _{m \rightarrow \infty} \mu_{m}(f)=\mu(f)$ for every $f \in \mathcal{C}_{\mathcal{P}}(X)$ ). Then, for every $k \in \mathbf{N}$,

$$
R_{\psi}(x)=\lim _{m \rightarrow \infty} R_{\varphi_{m}}(x)=\lim _{m \rightarrow \infty} \mu_{m}\left(\varphi_{m}\right) \leq \lim _{m \rightarrow \infty} \mu_{m}\left(\varphi_{k}\right)=\mu\left(\varphi_{k}\right) .
$$

Letting $k \rightarrow \infty$, we get $R_{\psi}(x) \leq \int \psi d \mu$. Trivially $\int \psi d \mu \leq \int R_{\psi} d \mu \leq R_{\psi}(x)$. Then

Corollary 3.5. Let $\psi: X \rightarrow[0, \infty]$ be upper semicontinuous and $\mathcal{P}$-bounded.

$$
M_{\psi}=N_{\psi}=R_{\psi}=\psi \vee \hat{R}_{\psi}
$$


Proof. By Proposition 3.4, $R_{\psi} \leq M_{\psi}$. By Proposition 2.4,(i), the nearly hyperharmonic function $u:=N_{\psi}=\psi \vee \hat{N}_{\psi}$ is Borel measurable, and hence, by Corollary 2.6, $M_{\psi} \leq M_{u} \leq u$. The proof is finished, since $u \leq R_{\psi}$, by (2.7).

For every $\varphi: X \rightarrow[0, \infty]$, let $\Psi_{\varphi}$ denote the set of all bounded upper semicontinuous functions $0 \leq \psi \leq \varphi$ with compact support in $\{\varphi>0\}$. We are now able to prove even more than announced in Theorem 3.1.

Theorem 3.6. Let $\varphi: X \rightarrow[0, \infty]$ be $\widetilde{\mathcal{B}^{*}}$-measurable. Then

$$
M_{\varphi}=\sup \left\{R_{\psi}: \psi \in \Psi_{\varphi}\right\}=N_{\varphi}=\varphi \vee \hat{N}_{\varphi}
$$

and there is an increasing sequence $\left(\psi_{m}\right)$ in $\Psi_{\varphi}$ such that

$$
N_{\varphi}=\varphi \vee \sup _{m \in \mathbf{N}} R_{\psi_{m}}=\varphi \vee \sup _{m \in \mathbf{N}} \hat{R}_{\psi_{m}}
$$

If $\varphi \leq s$ for some $s \in \mathcal{W} \cap \mathcal{C}(X)$, then $M_{\varphi}$ is harmonic on any open set, where $M_{\varphi} \geq \alpha \varphi$ for some $\alpha>1$.

Proof. Clearly, $M_{\varphi}=\sup \left\{M_{\psi}: \psi \in \Psi_{\varphi}\right\}$, where $M_{\psi}=N_{\psi}=R_{\psi}=\psi \vee \hat{R}_{\psi}$ for every $\psi \in \Psi_{\varphi}$, by Corollary 3.5. Since $(\varphi \wedge n) 1_{\{x\}} \in \Psi_{\varphi}, x \in X, n \in \mathbf{N}$, we obtain that

$$
M_{\varphi}=\sup \left\{R_{\psi}: \psi \in \Psi_{\varphi}\right\}=\varphi \vee \sup \left\{\hat{R}_{\psi}: \psi \in \Psi_{\varphi}\right\} \leq \varphi \vee \hat{N}_{\varphi}=N_{\varphi}
$$

In particular, $M_{\varphi}$ is $\widetilde{\mathcal{B}^{*}}$-measurable. By [4, I.1.7], there is an increasing sequence $\left(\psi_{m}\right)$ in $\Psi_{\varphi}$ such that $\sup _{m \in \mathbf{N}} \hat{R}_{\psi_{m}}=\sup \left\{\hat{R}_{\psi}: \psi \in \Psi_{\varphi}\right\}$. Then $\varphi \vee \hat{R}_{\psi_{m}} \uparrow M_{\varphi}$ as $m \rightarrow \infty$.

We now claim that

$$
M_{\varphi} \in \mathcal{N}^{+}
$$

and therefore $N_{\varphi} \leq M_{\varphi}$. Having (3.3) this implies that (3.1) and (3.2) hold.

So let $x \in V \in \mathcal{U}_{c}, \mu:=\varepsilon_{x}^{V^{c}}$. To show that $\int M_{\varphi} d \mu \leq M_{\varphi}(x)$ we may assume that $\int \varphi d \mu<\infty$, since otherwise $M_{\varphi}(x)=\infty$. Let $a<b<\int M_{\varphi} d \mu$. By (3.3), there exist $m \in \mathbf{N}$ and $\psi \in \Psi_{\varphi}$ such that

$$
b<\int \varphi \vee \hat{R}_{\psi_{m}} d \mu \quad \text { and } \quad \int(\varphi-\psi) d \mu<b-a .
$$

Of course, we may assume that $\psi_{m} \leq \psi$. Clearly, $\varphi \vee R_{\psi} \leq R_{\psi}+\varphi-\psi$. So

$$
b<\int \varphi \vee R_{\psi} d \mu \leq \int R_{\psi} d \mu+(b-a)
$$

and hence

$$
a<\int R_{\psi} d \mu \leq R_{\psi}(x) \leq M_{\varphi}(x)
$$

Finally, suppose that $\varphi \leq s$ for some $s \in \mathcal{W} \cap \mathcal{C}(X)$ and let $U$ be an open set, where $\varphi$ vanishes. Then all functions $g_{m}:=\left.R_{\psi_{m}}\right|_{U}$ are harmonic on $U$, by Proposition 3.3, and hence $\left.M_{\varphi}\right|_{U}=\sup g_{m}$ is harmonic on $U$. An application of the next Proposition completes the proof.

Proposition 3.7. Let $\mathcal{F}$ be a convex cone of numerical functions on a set $Y$ and $f_{0} \in \mathcal{F}, 0<f_{0}<\infty$. For every numerical function $\varphi \geq 0$ on $Y$, let

$$
F_{\varphi}:=\inf \{f \in \mathcal{F}: f \geq \varphi\} .
$$

Then $F_{\varphi 1_{X \backslash A}}=F_{\varphi}$ for every numerical function $\varphi \geq 0$ on $Y$ and every $A \subset X$ such that $\alpha \varphi \leq F_{\varphi} \wedge\left(M f_{0}\right)$ on $A$ for some $\alpha, M \in(1, \infty)$. 
Proof. Clearly, it suffices to consider the case, where $\varphi$ is not identically zero on $A$. Trivially, $u:=F_{\varphi 1_{X \backslash A}} \leq F_{\varphi}$. For the reverse inequality let $\varepsilon>0, v:=u+\varepsilon f_{0}$,

$$
\beta:=\inf \{b \in(0, \infty): \varphi \leq b v \text { on } A\} \quad \text { and } \quad \gamma:=1 \vee \beta .
$$

Then $0<\beta \leq M / \varepsilon$ and $\varphi \leq \gamma v$ on $X$. Hence $(\beta / \alpha) v(x)<\varphi(x)$ for some $x \in A$ and $F_{\varphi} \leq \gamma v$, which leads to $\beta v(x)<\alpha \varphi(x) \leq F_{\varphi}(x) \leq \gamma v(x)$. Thus $\gamma=1, v \geq F_{\varphi}$, and the proof is completed letting $\varepsilon \rightarrow 0$.

Remark 3.8. Suppose for a moment that every semipolar set is polar (axiom of polarity, Hunt's hypothesis $(\mathrm{H}))$ and let $\varphi: X \rightarrow[0, \infty]$ be $\tilde{\mathcal{B}}$-measurable. Then, by [15, Theorem 2.2] and (3.2), there exists an increasing sequence $\left(\psi_{n}\right)$ in $\Psi_{\varphi}$ such that

$$
R_{\varphi}=\varphi \vee \sup _{n \in \mathrm{N}} R_{\psi_{n}}=N_{\varphi}
$$

Let us note that the equality $R_{\varphi}=N_{\varphi}$ will follow as well from Proposition 5.3. Indeed, defining $u:=N_{\varphi}=\varphi \vee \hat{N}_{\varphi}$, we trivially have $R_{\varphi}=R_{u}$. By Proposition 5.3, the set $P:=\{\hat{u}<u\}$ is semipolar, hence polar because of hypothesis $(\mathrm{H})$. So we obtain that $R_{(u-\hat{u}) 1_{P}}=(u-\hat{u}) 1_{P}$ and therefore

$$
R_{u} \leq \hat{u}+R_{(u-\hat{u}) 1_{P}}=\hat{u}+(u-\hat{u}) 1_{P}=u \leq R_{u} .
$$

Thus $R_{u}=u, R_{\varphi}=N_{\varphi}$.

In fact, the equality $u=R_{u}(=\inf \{w \in \mathcal{W}: w \geq u\})$ holds for every nearly Borel measurable $u \in \mathcal{N}^{+}$even without assuming the axiom of polarity (see $[2$, Theorem 6.4] or [3, Theorem 4.3.3] based on long and subtle preparations).

\section{Application to Jensen measures}

In this section, let us suppose that $(X, \mathcal{W})$ is a harmonic space, that is, the harmonic measures $\mu_{x}^{V}=\varepsilon_{x}^{V^{c}}, V$ relatively compact open in $X, x \in X$, are supported by the boundary $\partial V$ of $V$.

Given an open set $U$ in $X$, let ${ }^{*} \mathcal{H}(U)$ denote the set of all hyperharmonic functions on $U$, that is, lower semicontinuous numerical functions $w>-\infty$ on $U$ such that $\int w d \varepsilon_{x}^{V^{c}} \leq w(x)$ for all open $V$, which are relatively compact in $U$, and $x \in V$.

Given $x \in U$, let $\mathcal{J}_{x}(U)$ denote the set of all Jensen measures for $x$ with respect to $U$, that is, measures $\mu$ with compact support in $U$ satisfying

$$
\int w d \mu \leq w(x) \text { for every } w \in^{*} \mathcal{H}(U) .
$$

In fact, it suffices to know (4.1) for all $w \in{ }^{*} \mathcal{H}(U) \cap \mathcal{C}(U)$, since every $w \in{ }^{*} \mathcal{H}(U)$ is an increasing limit of functions in ${ }^{*} \mathcal{H}(U) \cap \mathcal{C}(U)$.

Since $\mathcal{W}=\left\{w \in{ }^{*} \mathcal{H}(X): w \geq 0\right\}$ and $\left.{ }^{*} \mathcal{H}(X)\right|_{U} \subset{ }^{*} \mathcal{H}(U)$, we have

$$
\varepsilon_{x} \in \mathcal{J}_{x}(U) \subset \mathcal{J}_{x}(X) \subset \mathcal{M}_{x}(\mathcal{W}), \quad x \in U
$$

(where we consider measures in $\mathcal{J}_{x}(U)$ as measures on $X$ ). It will be convenient to introduce also the union $\mathcal{J}_{x}^{\prime}(X)$ of all $\mathcal{J}_{x}(U), U$ open relatively compact in $X, x \in U$ (see [13] for properties implying that $\mathcal{J}_{x}^{\prime}(X)=\mathcal{J}_{x}(X)$ ).

Finally, for every locally lower bounded function $\varphi$ on $X$ which is $\widetilde{\mathcal{B}^{*}}$-measurable, we define functions $J_{\varphi}$ and $J_{\varphi}^{\prime}$ on $X$ by

$$
J_{\varphi}(x):=\sup \left\{\int \varphi d \mu: \mu \in \mathcal{J}_{x}(X)\right\} \quad \text { and } \quad J_{\varphi}^{\prime}(x):=\sup \left\{\int \varphi d \mu: \mu \in \mathcal{J}_{x}^{\prime}(X)\right\} .
$$


If $\varphi \geq 0$, then obviously $M_{\varphi}^{\prime} \leq J_{\varphi}^{\prime} \leq J_{\varphi} \leq M_{\varphi}$. Therefore Proposition 2.5 and Theorem 3.6 immediately yield the following.

Theorem 4.1. Let $\varphi$ be a positive $\widetilde{\mathcal{B}^{*}}$-measurable numerical function on $X$. Then

$$
J_{\varphi}=J_{\varphi}^{\prime}=\varphi \vee M_{\varphi}^{\prime}=M_{\varphi}=N_{\varphi}=\varphi \vee \hat{N}_{\varphi}
$$

In particular, $J_{\varphi}$ is Borel measurable if $\varphi$ is Borel measurable.

Similarly as in [15] we may now extend this result to functions $\varphi$ which are not necessarily positive. To that end let $\mathcal{N}$ denote the set of all nearly hyperharmonic functions on $X$, that is, locally lower bounded functions $w: X \rightarrow]-\infty, \infty]$ such that $\int^{*} w d \varepsilon_{x}^{V^{c}} \leq w(x)$ for all $x \in X$ and relatively compact open neighborhoods $V$ of $x$. We immediately get the following generalization of Proposition 2.3.

Proposition 4.2. The set $\mathcal{N}$ of all nearly hyperharmonic functions on $X$ has the following properties:

(i) $\mathcal{N}$ is a convex cone containing ${ }^{*} \mathcal{H}(X)$.

(ii) For every $u \in \mathcal{N}, \hat{u}=\hat{u}^{f} \in{ }^{*} \mathcal{H}(X)$.

(iii) If $\left(u_{m}\right)$ is a sequence in $\mathcal{N}$ and $u_{m} \uparrow u$, then $u \in \mathcal{N}$ and $\hat{u}_{m} \uparrow \hat{u}$.

(iv) For every subset $\mathcal{V}$ of $\mathcal{N}$ which is locally lower bounded, inf $\mathcal{V} \in \mathcal{N}$.

Extending the definitions of $J_{\varphi}, J_{\varphi}^{\prime}$, and $M_{\varphi}^{\prime}$ in an obvious way, we get the following.

Corollary 4.3. Let $\varphi$ be a locally lower bounded $\widetilde{\mathcal{B}^{*}}$-measurable numerical function on $X$ such that $\varphi+h \geq 0$ for some harmonic function $h$ on $X$. Then

$$
J_{\varphi}=J_{\varphi}^{\prime}=\varphi \vee M_{\varphi}^{\prime}=N_{\varphi}=\varphi \vee \hat{N}_{\varphi} .
$$

In particular, $J_{\varphi}$ is Borel measurable if $\varphi$ is Borel measurable.

Proof. It suffices to observe that $\varphi+h$ is $\widetilde{\mathcal{B}^{*}}$-measurable and that obviously $J_{\varphi}=J_{\varphi+h}-h, J_{\varphi}^{\prime}=J_{\varphi+h}^{\prime}-h, M_{\varphi}^{\prime}=M_{\varphi+h}^{\prime}-h$, and $N_{\varphi}=N_{\varphi+h}-h$.

Localizing this result we may deal with functions $\varphi$ which are locally lower bounded.

Corollary 4.4. Let $\varphi$ be a locally lower bounded $\widetilde{\mathcal{B}^{*}}$-measurable numerical function on $X$ such that, for every relatively compact open set $U$ in $X$, there exists a harmonic function $h$ on $X$ with $\varphi+h \geq 0$ on $U$. Then

$$
J_{\varphi}^{\prime}=\varphi \vee M_{\varphi}^{\prime}=N_{\varphi}=\varphi \vee \hat{N}_{\varphi}
$$

In particular, $J_{\varphi}^{\prime}$ is Borel measurable if $\varphi$ is Borel measurable.

Proof. Let $U_{n}$ be relatively compact open sets in $X$ such that $U_{n} \uparrow X$ as $n \rightarrow \infty$. For every $n \in \mathbf{N}$, we apply Corollary 4.3 to the harmonic space $\left(U_{n},{ }^{*} \mathcal{H}^{+}\left(U_{n}\right)\right)$ and obtain that, for $x \in U_{n}$,

$$
\begin{aligned}
& \sup \left\{\int^{*} \varphi d \mu: \mu \in \mathcal{J}_{x}\left(U_{n}\right)\right\}=\varphi(x) \vee \sup \left\{\int^{*} \varphi d \varepsilon_{x}^{V^{c}}: x \in V \in \mathcal{U}_{c}, \bar{V} \subset U_{n}\right\} \\
& =\inf \left\{w(x): w \text { nearly hyperharmonic on } U_{n}, w \geq \varphi \text { on } U_{n}\right\}=: v_{n}(x),
\end{aligned}
$$

where $v_{n}(x)=\varphi(x) \vee \hat{v}_{n}(x)$. Defining $v_{n}(x):=\varphi(x), x \in X \backslash U_{n}$, we easily see that the sequence $\left(v_{n}\right)$ is increasing to a nearly hyperharmonic function $v$ on $X$, where $v=N_{\varphi}=\varphi \vee \hat{N}_{\varphi}$, by Proposition 4.2. The proof is completed letting $n \rightarrow \infty$. 
Remark 4.5. By Remark 3.8, the results in this Section imply the results in [15, Section 3].

\section{Some improvement of the measurability}

Let us now return to the general situation of an arbitrary balayage space $(X, \mathcal{W})$. Sometimes we can say a bit more about the measurability of $N_{\varphi}$ (and hence on the measurability of $J_{\varphi}$ in Section 4).

We recall that a set $A$ in $X$ is called thin at $x \in X$ if $\hat{\varepsilon}_{x}^{A} \neq \varepsilon_{x}$. It is totally thin if it is thin at every $x \in X$. Every totally thin set is finely closed and contained in a totally thin Borel set. A semipolar set is a countable union of totally thin sets.

So the $\sigma$-algebra $\mathcal{B}^{f}$ of all finely Borel subsets of $X$ (that is, the smallest $\sigma$-algebra on $X$ containing all finely open sets) contains all semipolar sets. By [4, VI.5.16]), for every $B \in \mathcal{B}^{f}$, there are $B_{1}, B_{2} \in \mathcal{B}$ such that $B_{1} \subset B \subset B_{2}$ and $B_{2} \backslash B_{1}$ is semipolar. Thus $\mathcal{B}^{f}$ is the smallest $\sigma$-algebra containing $\mathcal{B}$ and all semipolar sets. In particular, we have $\tilde{\mathcal{B}} \subset \mathcal{B}^{f}$.

Example 5.1. Suppose for the moment that $X=\mathbf{R}^{d} \times \mathbf{R}, d \geq 1$, and $\mathcal{W}$ is the set of all positive hyperharmonic functions associated with the heat equation on $\mathbf{R}^{d+1}$. Let $S$ be any subset of $\mathbf{R}^{d} \times\{0\}$. Then $S$ is semipolar; it is polar if and only if $S$ has outer $d$-dimensional Lebesgue measure zero. The function $u:=1_{S}+1_{\mathbf{R}^{d} \times(0, \infty)}$ is nearly hyperharmonic, $\mathcal{B}^{f}$-measurable, and $\{\hat{u}<u\}=S$.

Proposition 5.2. Let $S \in \widetilde{\mathcal{B}^{*}}$ be semipolar. Then there exists a sequence $\left(K_{n}\right)$ of compacts in $S$ such that the set $S \backslash \bigcup_{n=1}^{\infty} K_{n}$ is polar. In particular, $S \in \tilde{\mathcal{B}}$.

Proof. By [10, Theorem 1.5] (which holds as well for balayage spaces), there exists a measure $\mu$ on $X$ such that $\mu^{*}(B)>0$ for every subset $B$ of $S$ which is not polar. There exists a subset $S_{0} \in \mathcal{B}^{*}$ of $S$ such that the set $P_{0}:=S \backslash S_{0}$ is polar. Moreover, there exists a sequence $\left(K_{n}\right)$ of compacts in $S_{0}$ such that $P_{1}:=S_{0} \backslash \bigcup_{n=1}^{\infty} K_{n}$ is a $\mu$-null set, and hence polar. Since $P_{0} \cup P_{1}$ is polar, the proof is finished.

The equivalence in the following proposition is obvious if $u \in \mathcal{N}^{+}$satisfies

$$
u=R_{u},
$$

since then $u$ is finely upper semicontinuous and the set $\{\hat{u}<u\}$ is semipolar by [4, VI.5.11]. We recall from Remark 3.8 that (5.1) holds if $u \in \mathcal{N}^{+}$is nearly Borel measurable. It would be interesting to know if it is even satisfied if $u$ is only $\widetilde{\mathcal{B}^{*}}$ measurable.

Proposition 5.3. For every $u \in \mathcal{N}^{+}$the following statements are equivalent:

(i) The set $\{\hat{u}<u\}$ is semipolar.

(ii) The function $u$ is finely Borel measurable.

Proof. (i) $\Rightarrow$ (ii): For every $t>0$, the set $\{u \geq t\}$ is the union of $\{\hat{u} \geq t\} \in \mathcal{B}$ and the semipolar set $\{u \geq t>\hat{u}\}$.

(ii) $\Rightarrow$ (i): There is a semipolar Borel set $S_{0}$ such that the function $v_{0}:=u 1_{X \backslash S_{0}}$ is $\mathcal{B}$-measurable. Suppose that the set $\{\hat{u}<u\}$ is not semipolar. Then the Borel set $A:=\{\hat{u}<u\} \backslash S_{0}$ is not semipolar. So, by [4, VI.8.9], there is a measure $\mu \neq 0$ on $X$ such that $\mu(X \backslash A)=0$ and $\mu$ does not charge semipolar sets. There exist functions $\psi_{m} \in \Psi_{v_{0}}$ such that $\psi_{m} \uparrow v_{0}$ outside a $\mu$-null set $B \in \mathcal{B}$. By Corollary 3.5,

$$
\psi_{m} \leq R_{\psi_{m}}=N_{\psi_{m}} \leq N_{u}=u, \quad m \in \mathbf{N}
$$


Hence $\left\{\sup _{m \in \mathbf{N}} R_{\psi_{m}}<u\right\} \subset B \cup S_{0}$. Further, the union $S_{1}$ of all sets $\left\{\hat{R}_{\psi_{m}}<R_{\psi_{m}}\right\}$, $m \in \mathbf{N}$, is semipolar, and we obtain that

$$
\hat{u} \geq \sup _{m \in \mathbf{N}} \hat{R}_{\psi_{m}}=\sup _{m \in \mathbf{N}} R_{\psi_{m}}=u \quad \text { on } X \backslash\left(B \cup S_{0} \cup S_{1}\right) .
$$

Thus $A \subset B \cup S_{1}$ and $\mu(X)=\mu(A)=0$, a contradiction.

Corollary 5.4. If $\varphi: X \rightarrow[0, \infty]$ is $\mathcal{B}^{f} \cap \widetilde{\mathcal{B}^{*}}$-measurable, then the function $N_{\varphi}$ is $\widetilde{\mathcal{B}}$-measurable.

Proof. By Theorem 3.6, the function $u:=N_{\varphi}$ is $\mathcal{B}^{f} \cap \widetilde{\mathcal{B}^{*}}$-measurable. Let $t \in \mathbf{R}$ and $S:=\{u \geq t>\hat{u}\}$. Then $S \in \widetilde{\mathcal{B}^{*}}$ and $S$ is semipolar, by Proposition 5.3. So $S \in \widetilde{\mathcal{B}}$, by Proposition 5.2, and $\{u \geq t\}=\{\hat{u} \geq t\} \cup S \in \widetilde{\mathcal{B}}$.

Further, let $\mathcal{A}(X)$ denote the set of all numerical functions $\varphi$ on $X$ having the following property: For every $t \in \mathbf{R}$, there exists an analytic set $A$ in $X$ such that the set $\{\varphi \geq t\} \triangle A$ is semipolar. By the discussion preceding Proposition 5.3, $\varphi \in \mathcal{A}(X)$ for every finely Borel measurable function $\varphi$.

Proposition 5.5. Let $\varphi$ be a positive function in $\mathcal{A}(X)$ which is $\widetilde{\mathcal{B}^{*}}$-measurable. Then $N_{\varphi}$ is finely upper semicontinuous and $\widetilde{\mathcal{B}}$-measurable.

Proof. By Theorem 3.6, we know that $u:=N_{\varphi} \in \mathcal{A}(X)$ and $u$ is $\widetilde{\mathcal{B}^{*}}$-measurable. Let $x \in X$ and $u(x)<a$. We claim that $\{u<a\}$ is a fine neighborhood of $x$. Indeed, suppose the contrary. Then the set $A:=\{u \geq a\}$ is not thin at $x$. Let $A^{\prime}$ be an analytic set such that $A^{\prime} \subset A$ and $A \backslash A^{\prime}$ is semipolar. We fix $\eta \in(0,1)$ such that $u(x)<a \eta^{2}$. Since $V:=\left\{u_{0}(x)>\eta u_{0}\right\}$ is a neighborhood of $x$, we know, by [4, VI.4.2], that either the analytic set $A^{\prime} \cap V$ or the semipolar set $S:=\left(A \backslash A^{\prime}\right) \cap V$ is not thin at $x$.

If $A^{\prime} \cap V$ is not thin at $x$, then, by [4, VI.1.10 and 1.3.5], there is a compact $K$ in $A^{\prime} \cap V$ such that $R_{u_{0}}^{K}(x)>\eta u_{0}(x)$. By definition of semipolar sets, $S$ is the union of totally thin sets $T_{m}, m \in \mathbf{N}$. By [4, VI.5.7], the union of finitely many totally thin sets is totally thin. Hence we may assume without loss of generality that $T_{m} \uparrow S$ as $m \rightarrow \infty$. If $S$ is not thin at $x$, we then obtain, by [4, VI.1.7], that $R_{u_{0}}^{T_{m}}(x)>\eta u_{0}(x)$ for some $m \in \mathbf{N}$.

Thus, in any case, there exists a finely closed set $F$ such that

$$
F \subset A \cap V \in \widetilde{\mathcal{B}^{*}} \quad \text { and } \quad R_{u_{0}}^{F}(x)>\eta u_{0}(x) .
$$

Since $u \geq a>a \eta u_{0}(x)^{-1} u_{0}$ on $A \cap V$ and $\varepsilon_{x}^{F}(X \backslash(A \cap V))=0$, by [4, VI.4.6], we conclude that

$$
u(x) \geq \int u d \varepsilon_{x}^{F} \geq a \eta u_{0}(x)^{-1} \int u_{0} d \varepsilon_{x}^{F}=a \eta u_{0}(x)^{-1} R_{u_{0}}^{F}(x) \geq a \eta^{2}>u(x),
$$

a contradiction. By Corollary 5.4, the proof is finished.

Corollary 5.6. Let $\varphi: X \rightarrow[0, \infty]$ be such that, for every $t>0$, there exists an analytic set $A$ in $X$ such that the set $\{\varphi \geq t\} \triangle A$ is polar. Then $N_{\varphi}$ is $\widetilde{\mathcal{B}}$-measurable.

\section{References}

[1] Bauer, H.: Harmonische Räume und ihre Potentialtheorie. - Lecture Notes in Math. 22, Springer, Berlin, 1966.

[2] Beznea, L., and N. Boboc: Feyel's techniques on the supermedian functionals and strongly supermedian functions. - Potential Anal. 10:4, 1999, 347-372. 
[3] Beznea, L., and N. Boboc: Potential theory and right processes. - Mathematics and its Applications 572, Kluwer Academic Publishers, Dordrecht, 2004.

[4] Bliedtner, J., and W. Hansen: Potential theory - An analytic and probabilistic approach to balayage. - Universitext, Springer, Berlin-Heidelberg-New York-Tokyo, 1986.

[5] Cole, B. J., and T. J. RAnsford: Subharmonicity without semicontinuity. - J. Funct. Anal. $147,1997,420-442$.

[6] Constantinescu, C., and A. Cornea: Potential theory on harmonic spaces. - Grundlehren Math. Wiss., Springer, Berlin-Heidelberg-New York, 1972.

[7] Feyel, D.: Représentation des fonctionnelles surmédianes. - Z. Wahrsch. Verw. Gebiete 58:2, 1981, 183-198.

[8] Feyel, D.: Sur la théorie fine du potentiel. - Bull. Soc. Math. France 111:1, 1983, 41-57.

[9] Grigor'yan, A., and W. HAnsen: A Liouville property for Schrödinger operators. - Math. Ann. 312, 1998, 659-716.

[10] Hansen, W.: Semi-polar sets are almost negligible. - J. Reine Angew. Math. 314, 1980, 217220.

[11] Hansen, W.: Three views on potential theory. - A course at Charles University (Prague), Spring 2008, http://www.karlin.mff.cuni.cz/ hansen/lecture/course-07012009.pdf.

[12] Hansen, W., and I. Netuka: Harmonic measures for a point may form a square. - Adv. Math. 225, 2010, 1830-1839.

[13] Hansen, W., and I. Netuka: Jensen measures in potential theory. - Potential Anal. 37, 2012, $79-90$.

[14] Hansen, W., and I. Netuka: Unavoidable sets and harmonic measures living on small sets. - Proc. Lond. Math. Soc. 109, 2014, 1601-1629.

[15] Hansen, W., and I. Netuka: Reduced functions and Jensen measures. - Proc. Amer. Math. Soc. 146, 2018, 153-160.

[16] Mertens, J.-F.: Strongly supermedian functions and optimal stopping. - Z. Wahrscheinlichkeitstheorie Verw. Geb. 26, 1973, 119-139.

[17] Mоковоdzki. G.: Structure des cônes de potentiels. - Séminaire N. Bourbaki, exp. n³77, 1969-1970, 239-252.

[18] Mokobodzki, G.: Cônes de potentiels et noyaux subordonnés. - In: Potential Theory (C. I. M. E., I Ciclo, Stresa), Edizioni Cremonese, Rome, 1970, 207-248.

[19] Mokobodzki, G.: Ensembles compacts de fonctions fortement surmédianes. - In: Séminaire de Théorie du Potentiel, No. 4 (Paris, 1977/1978), Lecture Notes in Math. 713, Springer, Berlin, 1979, 178-193. 